\title{
The Development of International Standards for Very Small Enterprises
}

\author{
Claude Y. Laporte \\ École de technologie supérieure \\ Dept. of Software and IT Engineering \\ 1100, Notre-Dame Street West, Montréal, \\ Québec, Canada, H3C 1K3. \\ Claude.Y.Laporte@etsmtl.ca \\ +15143968956
}
Alain Renault
Centre de Recherche Public Henri Tudor
29, avenue John F. Kennedy
L-1855 Luxembourg-Kirchberg,
Luxembourg.
Alain.Renault@tudor.lu
+ 3524259 91-1

\author{
Simon Alexandre \\ Centre d'excellence en technologies de \\ l'information et de la communication \\ Rue Clement Ader, 8 \\ 6041 Charleroi, Belgium. \\ simon.alexandre@cetic.be \\ +32 71-919818
}

Kenneth V Crowder

Crowder \& Associates

$4424155^{\text {th }}$ Ave SE

Bellevue, WA 98006-2564, USA

kvcrowder@incose.org

+14256414187

Copyright (C 2008 by C. Y. Laporte, S. Alexandre, A. Renault \& K. Crowder. Published and used by INCOSE with permission.

\begin{abstract}
Industry recognizes that there are very small enterprises that develop parts which contain software components. These enterprises are very important to the world-wide economy, and the parts they develop are often integrated into products made by larger enterprises. Failure to deliver a quality product on time and within budget threatens the competitiveness of both organizations. One way to mitigate these risks is for all the suppliers in a product chain put in place recognized engineering practices. Many international standards have been developed to capture proven engineering practices. However, these standards were not written for very small development organizations, those with less than 25 employees, and are consequently difficult to apply in such settings. An ISO/IEC JTC1/SC7 ${ }^{1}$ Working Group has been established to address these difficulties.
\end{abstract}

\section{Introduction}

Today, the ability of organizations to compete, adapt, and survive depends increasingly on software. By 2010, it is estimated that a cellular phone, for example, will contain 20 million lines of code, and an automobile manufacturer has estimated that its cars will have up to 100 million lines of code by that time [Charette 2005]. Manufacturers depend increasingly on the components produced by their suppliers. A manufacturing chain of large mass-market products often has a pyramidal structure, as illustrated in Figure 1. For example, a large mass product manufacturer recently integrated into one of its products a part with an unknown software error that was produced by one of its 6,000 lower-level producers. This defective part resulted in a loss of over \$200 million by the mass product manufacturer.

\footnotetext{
${ }^{1}$ ISO/IEC JTC 1/SC7 stands for the International Organization for Standardization/International Electrotechnical Commission/Joint Technical Committee 1/Sub Committee 7, which is in charge of the development and maintenance of software and systems engineering standards.
} 


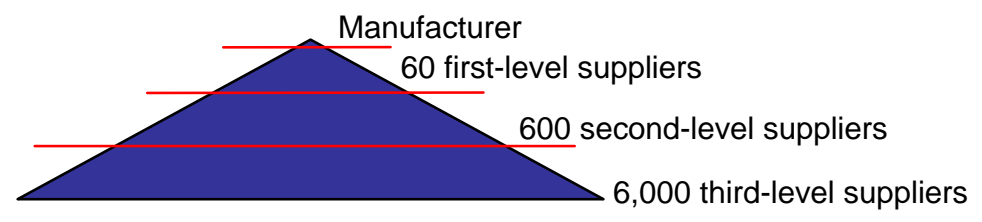

Figure 1 Example of the supply chain of a large manufacturer

Industry recognizes the value of VSEs (Very Small Enterprises) in contributing valuable products and services. In Europe, for instance, 85\% of the Information Technology (IT) sector's companies have 1 to 10 employees $^{2}$. In Canada, a recent survey of the Montreal area revealed, as illustrated in Table 1, that $78 \%$ of software development enterprises have fewer than 25 employees and 50\% have fewer than 10 employees [Laporte 2005]. In Brazil, small IT companies represent about $70 \%$ of the total number of companies [Anaclecto 2004].

Table 1 Size of software development companies in the Montreal area

\begin{tabular}{|c||c|c|c|c|}
\hline \multirow{2}{*}{$\begin{array}{c}\text { Size } \\
\text { (number of } \\
\text { employees) }\end{array}$} & \multicolumn{2}{c|}{$\begin{array}{c}\text { Software } \\
\text { Enterprises }\end{array}$} & \multicolumn{2}{c|}{ Jobs } \\
\cline { 2 - 5 } & Number & $\mathbf{\%}$ & Number & $\mathbf{\%}$ \\
\hline \hline 1 to 25 & 540 & $78 \%$ & 5,105 & $29 \%$ \\
\hline 26 to 100 & 127 & $18 \%$ & 6,221 & $36 \%$ \\
\hline over 100 & 26 & $4 \%$ & 6,056 & $35 \%$ \\
\hline \hline TOTAL & $\mathbf{6 9 3}$ & $\mathbf{1 0 0 \%}$ & $\mathbf{1 7 , 3 8 2}$ & $\mathbf{1 0 0 \%}$ \\
\hline
\end{tabular}

There is a need to help these organizations understand the benefit of the concepts, processes, and practices described in the ISO/IEC JTC1/SC7's international software engineering standards, and initiate their use. This paper describes a new project formed to facilitate access to, and utilization of, ISO/IEC JTC1/SC7 software engineering standards by VSEs with fewer than 25 employees. This paper is divided into six sections. The remaining sections include:

- a history of the events that led to an ISO/IEC JTC1 SC7 Project Proposal for Very Small Enterprises (VSEs);

- results of a world-wide survey of VSEs;

- an overview of the approach and processes used by Working Group 24 to produce an initial profile, guides, and templates;

- results from recent WG24 meetings;

- the direction of future work of WG24.

\section{History leading to an ISO/IEC Working Group for VSEs}

The mandate of ISO/IEC JTC1/SC7 is the standardization of processes, supporting tools, and supporting technologies for the engineering of software products and systems. A description of SC7 and of the development of ISO/IEC JTC1/SC7 standards is presented in [Coallier 2003]. In this section, we provide a brief history of the events leading to the creation of a new ISO/IEC

\footnotetext{
${ }^{2}$ http://www.esi.es/en/main/iitmark.html
} 
JTC1/SC7 Working Group (WG). A detailed description of its history is available in [Laporte 2006, Laporte 2008].

At the May 2004 SC7 Plenary meeting in Brisbane, Australia, Canada raised the issue of small enterprises requiring standards adapted to their size and maturity level. At that time, software engineering standards targeted (or were perceived as targeting) large organizations. A meeting of interested parties was organized and a consensus was reached on general objectives for a future working group to:

- make the current software engineering standards more accessible to VSEs;

- provide documentation requiring minimal tailoring and adaptation effort;

- provide harmonized documentation integrating available standards;

- produce process standards;

- identify work products and deliverables;

- support process and product assessment and quality;

- identify modeling techniques and tools;

- align profiles, if desirable, with the notions of capability levels presented in ISO/IEC 15504.

In March 2005, the Thailand Industrial Standards Institute (TISI) invited a Special Working Group (SWG) to advance the work items defined at the Brisbane meeting. A key topic of discussion was the clear definition of the size of VSEs that the SWG would target for IT services. The consensus was that they would focus on organizations and projects with 1 to 25 employees. The major output of this one-week meeting was a draft of the New Work Item (NWI) to be presented at the next SC7 meeting.

In May 2005, a resolution was approved to distribute for ballot the NWI Proposal for the development of Software Life Cycle Profiles and Guidelines for Use in Very Small Enterprises. Twelve countries voted in favor of the NWI Proposal [NWI 2005]. As a result of this vote, the project was approved and the new working group, WG24, was established. The leaders who were appointed to WG24 were:

- Mr. Tanin Uthayanaka (Thailand) - Convener;

- Mr. Claude Y. Laporte (IEEE Computer Society) - Project Editor;

- Mr. Jean Bérubé (Canada) - Secretary.

The Thailand Industrial Standards Institute (TISI) sent out a second invitation to participate in the SWG, to be held in September 2005 in Bangkok. The main objective of the meeting was to prepare material which would be presented to WG24 in order to facilitate the start-up of the working group that was scheduled for the Interim SC7 meeting in October 2005.

In October 2005, Italy hosted the ISO/IEC JTC1 SC7 Interim Meeting. The NWI was updated in order to take into account relevant comments received during balloting, and WG members validated the requirements. In addition, some VSE Business Models were identified, as was a strategy for creating profiles. Finally, WG24 decided to conduct a survey to collect relevant information from VSEs around the world.

WG24 met in Thailand, during the SC7 Plenary meeting in May 2006, with two new countries, India and Mexico, sending delegates. The main outputs of the meeting were:

- analysis of the survey responses;

- evaluation of documents tabled by national delegations;

- a decision to accept an existing Mexican standard described in the WG24 Approach (below) as initial input for the development of VSE profiles, guides, and templates. 


\section{Presentation of the Survey of VSEs}

The WG24 survey was developed to question VSEs about their use of ISO/SC7 standards, as well as to collect data to identify problems and potential solutions to help them apply the standards and become more accurate and comprehensive in developing their products. From the very beginning, the working group drew up several working hypotheses regarding VSEs. This survey was intended to determine which of these hypotheses were applicable, for example:

- The VSE context requires light and well-focused life cycle profiles.

- Particular business contexts require particular profiles.

- There are significant differences, in terms of available resources and infrastructure, between a VSE employing 1 to 10 people and an Information Technology (IT) department of the same size in a larger company.

- VSEs are limited in terms of both time and resources, which leads to a lack of understanding of how to use the standards for their benefit.

- Benefits for VSEs may include recognition through assessment or audit by an accredited body.

The survey questionnaire and an introductory text were developed by WG24 and translated into 9 languages: English, French, German, Korean, Portuguese, Thai, Turkish, Russian, and Spanish. The survey is made up of 20 questions structured in 5 parts: General information; Information about standards utilization in VSEs; Information about implementation and assessment problems in VSEs; Information about VSE needs; and Information about justification for compliance to standard(s).

A Web site, hosted by the École de Technologie Supérieure (ÉTS), was developed to minimize the difficulties in completing the survey and maximize the number of responses, and to facilitate data collection and the analysis of the results. A mailing list was created using WG24 members' contact networks. Also, professional centers and software engineering professors specializing in small software enterprises were contacted to encourage VSE participation.

Respondents were informed that it would take a maximum of 15 minutes to complete the survey. They were also informed that all data would be kept confidential, and that only summary results and project data that could not be matched to a specific VSE would be included in the published results.

In order to increase participation in the survey, WG24 promised to send all respondents a report presenting, anonymously, the survey results. The survey was launched in February 2006, and, as of June 2006, 392 responses had been collected from 29 countries.

Categorization of the sample according to the size criterion. In order to avoid developing profiles which would not meet the needs of VSEs, WG24 defined what VSEs are in terms of size. At the time, there was no official definition of the VSE, while the concept of the small and medium-sized enterprise (SME) was already clearly defined in Europe (fewer than 250 employees or with a turnover $\leq € 50$ million) and in the United States (fewer than 500 employees). The Organization for Economic Co-operation and Development (OECD) subdivides the SME category into several subcategories: micro (0-9 employees ${ }^{3}$ ); small (10-49 employees); and medium-sized (50-250 or 500 in USA). In Europe, Micro enterprises represent $93 \%$ of the total number of companies (56\% in USA) and 66\% of the total employment.

\footnotetext{
${ }^{3}$ A zero-employee company is one formed by its founder without any additional employees (e.g. a consultant).
} 
Of the 392 responders, 228 are enterprises with 0 to 25 employees (58\%), as illustrated in Figure 2. Note that responders representing small organizations ( $<25$ persons) which are part of a larger enterprise are not included among these 228 responses. These 228 VSEs constitute the sample for this study. The following paragraphs present findings common to the 228 VSEs and identify correlations within the sample, and findings which differ from those of the bigger companies that took part in the survey.

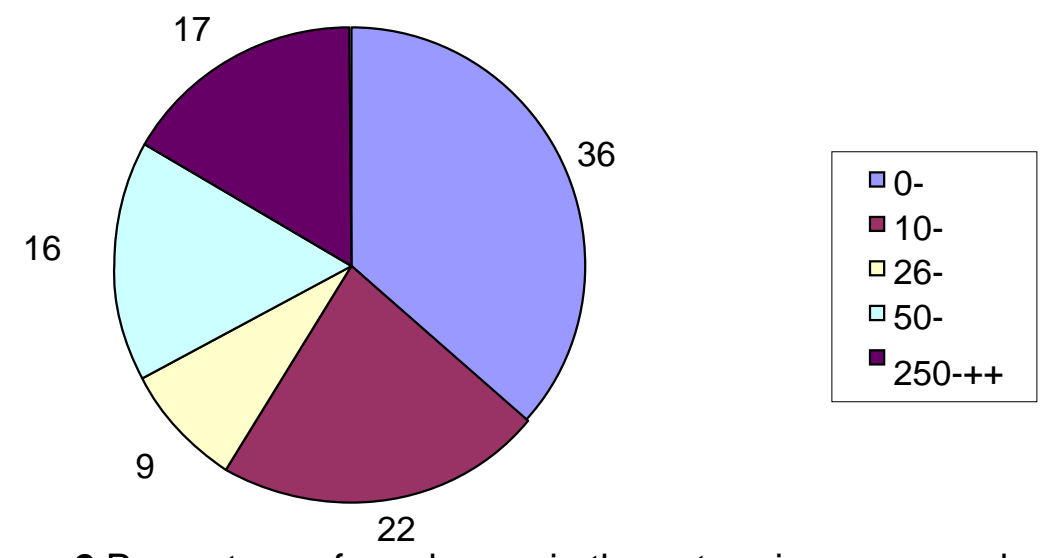

Figure 2 Percentage of employees in the enterprises surveyed

This categorization and several studies underscore the differences between micro, small, and medium-sized enterprises [Laporte 2006] in terms of available resources. Therefore, WG24 decided to focus on the first category (micro enterprises with 0-9 employees) and on a subgroup of the small enterprise category (10-25 employees).

General characteristics. Here, we draw attention to some weaknesses of the sample itself. The fact that the survey was initiated through WG24 contacts without building a true random sample may have impacted the survey results. The most obvious feature of the respondent sample, as illustrated in Table 2, is the geographical distribution of the respondents. We collected a high number of responses from Latin America (46\%), mainly from Colombia (22\%) and Brazil $(17 \%)$.

Table 2 Number of survey responses per country

\begin{tabular}{|l|c|l|c|}
\hline \multicolumn{1}{|c|}{ Country } & $\begin{array}{c}\text { Number } \\
\text { of } \\
\text { Responses }\end{array}$ & \multicolumn{1}{|c|}{ Country } & $\begin{array}{c}\text { Number } \\
\text { of } \\
\text { Responses }\end{array}$ \\
\hline Argentina & 2 & Italy & 2 \\
\hline Australia & 10 & Japan & 3 \\
\hline Belgium & 10 & South Korea & 4 \\
\hline Brazil & 70 & Luxembourg & 2 \\
\hline Bulgaria & 3 & Mexico & 20 \\
\hline Canada & 9 & New Zealand & 1 \\
\hline Chile & 1 & Peru & 4 \\
\hline Colombia & 109 & Russia & 4 \\
\hline Czech Republic & 3 & South Africa & 10 \\
\hline Dominican Republic & 1 & Spain & 3 \\
\hline Ecuador & 9 & Taiwan & 1 \\
\hline
\end{tabular}




\begin{tabular}{|l|c|l|c|}
\hline Finland & 13 & Thailand & 58 \\
\hline France & 4 & Turkey & 1 \\
\hline Germany & 1 & United Kingdom & 2 \\
\hline India & 57 & United States & 3 \\
\hline Ireland & 10 & \multicolumn{2}{|l}{} \\
\hline
\end{tabular}

At the same time, we received only a few responses from European countries (48), Japan (3), and the United States (3). The reasons for this are the following:

- The invitation to participate in the survey was not distributed in some countries.

- Many SPIN (Software Process Improvement Network) members are employed in larger companies not directly targeted by this survey.

- Most SPIN members already use CMMI, and they may not be interested in ISO standards.

- Most VSEs do not care about IT standardization, so only those aware of it or interested in it took the time to contribute.

Therefore, our results might only generalize to the broader populations of projects in each region to the extent that this sample represents them. Moreover, we have no evidence that participating companies are representative of the situation in their own countries.

Features of the VSE results. Among the respondents, the majority (79\%) are private companies and 78\% operate at their national level only. VSEs were asked to select their application domains. As shown in Figure 3, 93 respondents (over 40\%) are developing software for life-/mission-critical systems and 79 (34\%) on regulated developments. This underscores our hypothesis concerning the awareness of the participating companies, as it is assumed that companies working in these particular contexts are prone to using standards for contractual reasons.

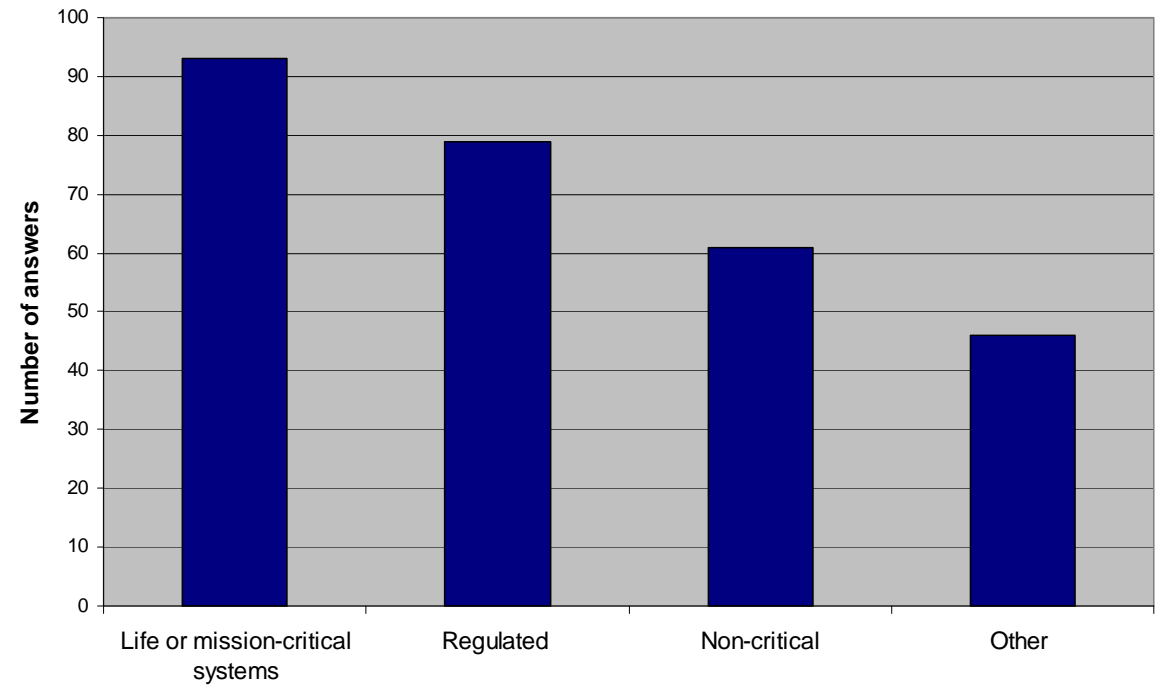

Figure 3 Application domains 
With regard to the types of software development, a large majority involve customized (tailored) software and specialized products, as shown in Figure 4.

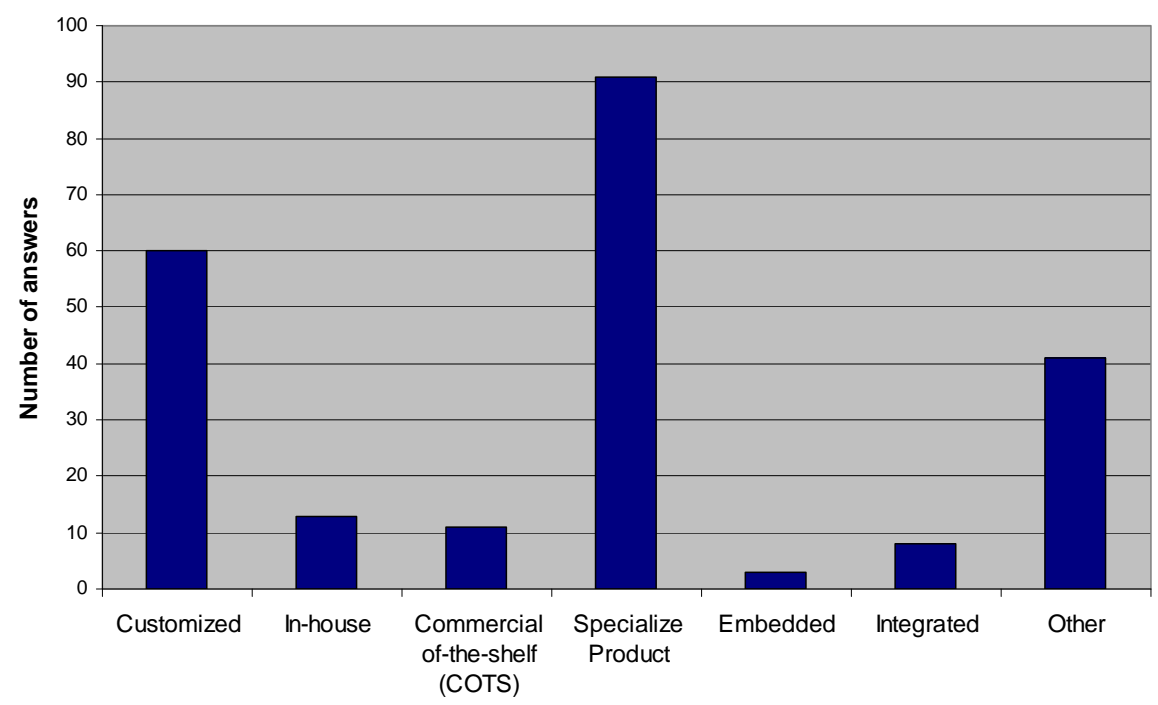

Figure 4 Types of software development

Use of Standards. An interesting finding of the survey is the difference in the percentage of certified companies with regard to company size: less than $18 \%$ of the VSEs are certified, while $53 \%$ of larger companies (more than 25 employees) claim to be certified. Furthermore, among the $18 \%$ not certified, $75 \%$ do not use standards.

WG24 anticipated the weak use of standards by VSEs by asking questions designed to provide a better understanding of the reasons for this. There are three main ones, as shown in Figure 5. The first is a lack of resources (28\%); the second is that standards are not required (24\%); and the third derives from the nature of the standards themselves: $15 \%$ of the respondents consider that the standards are difficult and bureaucratic, and do not provide adequate guidance for use in a small business environment.
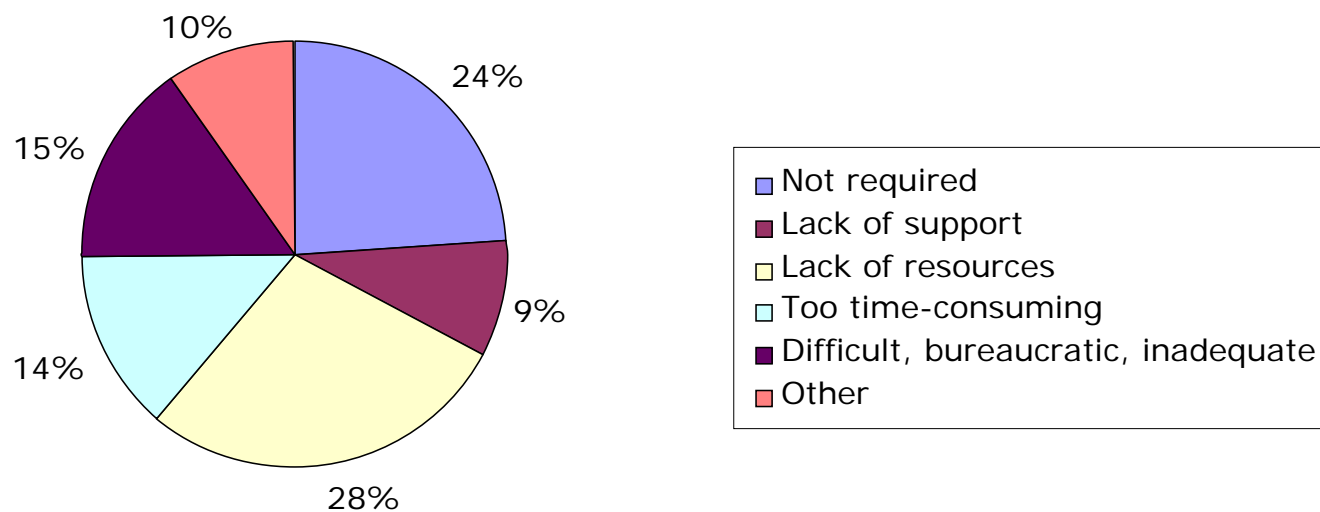

Figure 5 Why don't VSEs use standards? 
For a large majority (74\%) of VSEs, it is very important to be evaluated or certified against a standard. ISO certification is requested by $40 \%$ of them. Of the $28 \%$ requesting official market recognition, only $4 \%$ are interested in a national certification. From the VSE perspective, some benefits provided by certification are:

- increased competitiveness,

- greater customer confidence and satisfaction,

- greater software product quality,

- increased sponsorship for process improvement,

- decreased development risk,

- facilitation of marketing (e.g. a better image),

- higher potential for export.

However, VSEs are expressing the need for assistance in adopting and implementing standards. Over 62\% would like more guidance with examples, and 55\% are asking for lightweight and easy-to-understand standards, complete with templates. Finally, the respondents have indicated that it has to be possible to implement standards with minimum cost, time, and resources. All data about VSEs and standards clearly confirm WG24's hypothesis and the requirements. Therefore, WG24 is using this information for the development of profiles, guides, and templates to meet VSE needs.

\section{The WG 24 Approach}

The approach used by WG24 had to take into account, as a starting point, the ISO requirements in terms of the definition of a standard. Indeed, since an international standard dedicated to the software life cycle was already available (i.e. ISO/IEC 12207)[ISO 2007], WG24 had to use the concept of ISO profiles (ISP - International Standardized Profile) to develop the new standard for VSEs. A profile is defined as "A set of one or more base standards and/or ISPs, and, where applicable, the identification of chosen classes, conforming subsets, options and parameters of those base standards, or ISPs necessary to accomplish a particular function" [ISO 1998]. From a practical point of view, a profile is a kind of matrix which identifies precisely all elements that are taken from existing standards from those that are not. This prevents the new standard of copying the content of the existing ISO/IEC 12207 standard, but allows to the highlighting of some clauses if and when appropriate.

The overall approach followed by WG24 to develop this new standard for VSE consisted of three steps:

- Select the ISO/IEC12207 process subset applicable to VSEs with fewer than 10 employees

- Tailor the subset to fit VSE needs

- Develop guidelines

First, since WG24 wished to prepare an initial set of software development standards as quickly as possible, WG24 analyzed international reference standards and models which could help tailor subset ISO/IEC 12207 for low maturity VSEs. To create these initial products quickly, WG24 began a search for existing standards or models which could be tailored. MoProSoft, a Mexican standard developed to assist Mexican small and medium-sized enterprises (SMEs), was selected in order to achieve this objective [NMX 2005].

MoProSoft uses ISO/IEC 12207 as a general framework. It borrows practices from ISO9001, Capability Maturity Model Integration (CMMI) ${ }^{\circledR}$ developed by the Software Engineering 
Institute [SEI 2006], the Project Management Body of Knowledge (PMBOK) [IEEE 2003], and the Software Engineering Body of Knowledge SWEBOK [Abran 2004].

However, WG24 felt that MoProSoft addressed the needs of organizations larger than the targeted VSEs. Therefore, in a second step, WG24 decided to tailor MoProSoft to address key characteristics of low maturity VSEs. The tailoring approach led to the development of incremental profile targeting as a starting point, low maturity VSEs with fewer than 10 employees, and, in a second phase, those with 10 to 25 employees. Therefore, the first profile developed by WG24 contains basic activities from project management- and software development-related processes. The idea was to concentrate on core activities which a lowmaturity VSE should perform.

The third step of the approach consisted of defining guidelines explaining in more detail the processes defined in the profile. These guidelines will be published as ISO Technical Reports, which should be freely accessible to VSEs. These guidelines integrate a series of deployment packages. A deployment package is a set of artifacts developed to facilitate the implementation of a set of practices of the selected framework in a VSE. The elements of a typical deployment package are: process description (e.g. activities, inputs, outputs, and roles), guide, template, checklist, example, presentation material, reference and mapping to standards and models, and a list of tools. Packages are designed such that a VSE can implement its content without having to implement the complete framework at the same time. The table of contents of a deployment package is illustrated in Table 3.

Table 3 Table of contents in a deployment package.

\begin{tabular}{|l|}
\hline 1. Introduction \\
\hline Purpose of this document \\
\hline Key Definitions \\
\hline 2. Why this Process is important \\
\hline 3. Overview of Main Tasks \\
\hline 3.1 Tasks \\
\hline 3.2 Roles and artifacts \\
\hline 3.3 Activity Lifecycle and examples of lifecycles \\
\hline Appendix A Templates \\
\hline Appendix B Checklists \\
\hline $\begin{array}{l}\text { Appendix C Coverage Matrices (ISO 12207, ISO 9001, } \\
\text { CMMI) }\end{array}$ \\
\hline Appendix D Tools \\
\hline Appendix E Training Material \\
\hline Appendix F Deployment Package Evaluation Form \\
\hline
\end{tabular}

\section{Recent Developments}

At the Montreal meeting of WG24 in October 2007, the requirement analysis and management deployment package was reviewed and received broad support from the group members. The group decided to develop additional deployment packages for its Berlin meeting in May 2008: change management, project management, and testing.

Having profiles and guides for VSEs is not sufficient to ensure broad utilization and adoption, however. They have to be tested with real VSEs in a few countries. The Mexican 
delegation presented the results of the introduction of the first profile developed by WG24 as a pilot project, in a number of Latin American countries [Oktaba 2007]. Also, a new country, Columbia, and a new organization, the European Software Institute (ESI), joined WG24.

The delegate from Columbia works as the quality assurance director of the Parquesoft Foundation ${ }^{4}$. Parquesoft, a non-profit organization, houses over 250 VSEs and more than 1,000 software engineering professionals. In one location, Cali, there are over 125 VSEs under the same roof. Such a setting will facilitate the piloting of deployment packages in many VSEs under the supervision of its quality assurance director.

\section{Conclusion and Future Work}

Industry recognizes the contribution of VSEs in terms of the valuable products and services they offer. About 75\% of software enterprises worldwide have fewer than 25 employees. The current collection of ISO/IEC JTC1 SC7 standards is not easily applied in VSEs, which generally find standards difficult to understand. WG24, established by JTC1/SC7 to address this issue, conducted an international survey of VSEs in order to refine the requirements related to the design of a software engineering standards for this type of company. Results of the survey mainly underscore the VSE's need for guidance and practical support material to implement standards. Taking into account these findings, WG24 started implementation of a reference model based on a Mexican national standard developed for SMEs.

With regard to future work, WG24 plans to invite VSEs, especially those that responded to the survey, to participate in the field trials. Since a few WG24 delegates are already working closely with VSEs, they will play a key role in coordinating the trials. These trials will help to validate the approach and obtain feedback in order to improve the documents before they go to ISO/IEC publication. Profiles and guides, such as the assessment guide and the management and engineering guide, will also be circulated by the ISO for review and ballot in 2008. WG24 is planning to produce a Final Draft in 2009. Publication by the ISO/IEC is scheduled for 2010. In the meantime, deployment packages will be made available to VSEs on public Web sites.

Additional Information. The following Web sites provide more information, as well as articles by WG24 members (and eventually deployment packages developed by them):

- $\quad$ http://profs.logti.etsmtl.ca/claporte/English/VSE/index.html

- http://www.cetic.be/indexEN.php3

\section{References}

Abran, A., Moore, J. W., Bourque, P., and Dupuis, R. (eds.). Guide to the Software Engineering Body of Knowledge. IEEE Computer Society Press, 2004.

Anacleto, A., von Wangenheim, C. G.; Salviano, C. F.; Savi, R. Experiences gained from applying ISO/IEC 15504 to small software companies in Brazil, 4th International SPICE Conference on Process Assessment and Improvement, Lisbon, Portugal, April 2004, pp. 3337.

Charette, R. N., Why Software Fails, IEEE Computer Society, Spectrum, September 2005, pp 42-49.

\footnotetext{
${ }^{4}$ http://www.parquesoft.com
} 
Coallier, F., International Standardization in Software and Systems Engineering, Crosstalk, February 2003, pp. 18-22.

Friedman, Thomas L., The World is Flat, Farrar, Straus and Giroux; New York, New York; 2005.

(IEEE) IEEE 1490-2003, IEEE Guide Adoption of PMI Standard A Guide to the Project Management Body of Knowledge (PMBOK), 2003.

(ISO) ISO/IEC TR 10000-1:1998, Information technology — Framework and taxonomy of International Standardized Profiles - Part 1: General principles and documentation framework.

(ISO) ISO/IEC 12207, Information technology - Software life cycle processes. International Organization for Standardization/International Electrotechnical Commission: Geneva, Switzerland.

(ISO) ISO 9001:2000 Quality Management System Requirements, International Organization for Standardization.

Laporte, C. Y., Renault, A., Desharnais, J. M., Habra, N., Abou El Fattah, M., Bamba, J. C, Initiating Software Process Improvement in Small Enterprises: Experiment with MicroEvaluation Framework, SWDC-REK, International Conference on Software Development, University of Iceland, Reykjavik, Iceland, May 27-June 1, 2005, pp. 153-163.

Laporte, C. Y., April, A., Applying Software Engineering Standards in Small Settings: Recent Historical Perspectives and Initial Achievements. In: Proceedings of the First International Research Workshop for Process Improvement in Small Settings. Software Engineering Institute, Carnegie Mellon University, CMU/SEI-2006-Special Report-001, January 2006, pp. 39-51.

Laporte, C. Y., Renault, A., Alexandre, S., Applying ISO/IEC Software Engineering Standards in Very Small Enterprises, in Software Process Improvement for Small and Medium Enterprises: Techniques and Case Studies, Idea Group Inc, Hershey, PA, 2008.

(NMX) NMX-059-NYCE-2005, Information Technology-Software-Models of Processes and Assessment for Software Development and Maintenance. Part 01: Definition of Concepts and Products; Part 02: Process Requirements (MoProSoft); Part 03: Guidelines for Process Implementation; Part 04: Guidelines for Process Assessment (EvalProSoft), Ministry of Economy, Mexico, 2005.

(NWI) New Work Item Proposal - Software Life Cycles for Very Small Enterprises, ISO/IEC JTC1/SC7 N3288, May 2005. http://www.jtc1-sc7.org/.

Oktaba, H., Garcia, F., Piattini, M., Ruiz, F., Pino, F. J., Alquicira, C., Software Process Improvement: The Competisoft Project, IEEE Computer, October 2007, Vol. 40, No 10, pp. 21-28

(SEI) Software Engineering Institute. (2006). Capability Maturity Model Integration (CMMI). Pittsburgh PA: Carnegie Mellon University. Version 1.2, CMU/SEI-2006-TR-008. 


\section{About the Authors}

Claude Y Laporte is a professor at the École de Technologie Supérieure (ÉTS), an engineering school, where he teaches graduate and undergraduate courses in software engineering. His research interests include software process improvement in small and very small companies, and software quality assurance. He received a Master's degree in physics from the Université de Montréal and a Master's degree in applied sciences from the École Polytechnique de Montréal. He is the editor of an ISO/IEC-JTC1 SC7 working group tasked to develop software life cycle profiles and guidelines for use in very small enterprises. He is a member of IEEE, PMI, INCOSE and a member of the Professional Association of Engineers of the Province of Québec. He can be contacted at the École de technologie supérieure, Department of Software and IT Engineering, 1100 rue Notre-Dame Ouest, Montréal, Québec, Canada, H3C 1K3; Claude.Y.Laporte@etsmtl.ca.

Simon Alexandre is currently in charge of the Software \& System Engineering R\&D Department at CETIC, an ICT research center in Belgium. He has been a scientific collaborator in the software engineering department of the Computer Institute of the University of Namur since 2001. His interests are software process assessment and improvement techniques for small and medium enterprises, agile methodologies, automation of software product quality analysis, and Open Source development processes. He received his MS degree in informatics from the University of Namur. He is a member of the ISO/IEC-SC7 Working Group 24, which is tasked to develop profiles and guidelines for software life cycle use in very small enterprises. He can be contacted at CETIC, Clement Ader 8, 6041 Gosselies, Belgium; simon.alexandre@cetic.be.

Alain Renault is a project leader at the Public Research Center Henri Tudor - Luxembourg. He is a 1984 software engineering graduate. After 9 years in the industry as a software engineer, he returned to university, where he contributed to the development of OWPL (SPI framework \& Micro-Evaluation for SME). He has been working on SME projects for the past 8 years, recently focusing on security and service management. He is a member of an ISO/IEC-SC7 working group tasked to develop software life cycle profiles and guidelines for use in very small enterprises. Alain is also co-editor of a ISO/SC7 new work item proposal aiming to develop an incremental conformity to ISO/IEC 20000-1 on IT Service Management. He can be contacted at the Centre de Recherche Public Henri Tudor, 29, avenue J F Kennedy, L-1855 LuxembourgKirchberg, Luxembourg; alain.renault@tudor.lu.

Kenneth V. Crowder retired after 40+ years of successfully completing engineering and management assignments in the United States and internationally. He has extensive program and proposal experience in aerospace, education, contracting, and finance, and is supporting selected clients by providing consultation for program management, proposal development, product and software development, and capability maturity. Mr. Crowder currently is the principal and owner of Crowder and Associates, the head of delegation for INCOSE (International Council On Systems Engineering) liaison to ISO/IEC, member of multiple ISO/IEC JTC1 SC7 working groups, and a visiting executive for the IESC (International Executive Service Corps). He can be contacted at kenneth.crowder@incose.org. 